\title{
Modeling and Solution of Economic Dispatch Problem for GTCC Units
}

\author{
Yongmei Wang $^{1 *}$, Hua Xiao ${ }^{2 *}$ \\ ${ }^{1}$ College of Information Engineering, Zhengzhou University, Zhengzhou, China \\ ${ }^{2}$ College of Electric Power, South China University of Technology, Guangzhou, China \\ Email: ieymwang@zzu.edu.cn,xhua2008@163.com
}

Received February, 2013

\begin{abstract}
Economic dispatch problem lies at the kernel among different issues in GTCC units' operation, which is about minimizing the fuel consumption for a period of operation so as to accomplish optimal load dispatch among units. This paper has analyzed the load dispatch model of gas turbine combined-cycle (GTCC) units and utilizes a quantum genetic algorithm to optimize the solution of the model. The performance of gas turbine combined-cycle units varies with many factors and this directly leads to variation of model parameters. To solve the dispatch problem, variable constraints are adopted to correct the parameters influenced by ambient conditions. In the simulation, comparison of dispatch models for GTCC units considering and not considering the influence of ambient conditions indicates that it is necessary to adopt variable constraints for the dispatch model of GTCC units. To optimize the solution of the model, a Quantum Genetic Algorithm is used considering its advantages in searching performance. QGA combines the quantum theory with evolutionary theory of genetic algorithm. It is a new kind of intelligence algorithm which has been successfully employed in optimization problems. Utilizing quantum code, quantum gate and so on, QGA shows flexibility, high convergent rate, and global optimal capacity and so on. Simulations were performed by building up models and optimizing the solutions of the models by QGA. QGA shows better effect than equal micro incremental method used in the previous literature. The operational economy is proved by the results obtained by QGA. It can be concluded that QGA is quite effective in optimizing economic dispatch problem of GTCC units.
\end{abstract}

Keywords: GTCC Units; Economic Dispatch; Variable Constraints; Quantum Genetic Algorithm

\section{Introduction}

Due to the availability of natural gas and the advantages [1,2] of GTCC units, GTCC plants continue to gain strength in power industry. In this situation, economic operation of the GTCC units becomes much important. Economic dispatch problem lies at the kernel among different issues in GTCC units' operation [3, 4].

The economic dispatch problem is about minimizing the fuel cost for a period of operation so as to accomplish optimal load dispatch among units and in return satisfying the total load demand and operation constraints. Modeling of the load dispatch problem is necessary for the calculation of the problem. In practical, performance of a GTCC unit varies with many factors [5-7]. And this will lead to variation of load dispatch model. Environmental factors are uncontrollable and always changing with time and location. So the influence of environmental conditions on establishing the economic dispatch model is analyzed.

\footnotetext{
*These authors contributed equally to this work.
}

Mathematically, the problem of economic dispatch is a complex nonlinear problem containing integer and continuous variables. Many efforts [8-10] have been made to solve the problem, through various mathematical programming and optimization techniques. But these methods all have certain limitations such as requiring the formulation in continuous differentiable form, high computation time, failing to provide global optimal solution and so on.

With the development of computer technology and artificial intelligence, modern intelligent algorithms [11-14] show great advantages in solving economic dispatch problem. Quantum genetic algorithm (QGA) is a searching probabilistic method which combines the quantum theory with evolutionary theory of genetic algorithm. It utilizes the methods of quantum technology to improve the diversity of genetic algorithms' coding so that the algorithm will have faster calculation speed and stronger global optimization ability. Nowadays, quantum genetic algorithm has been widely applied to combination optimization problems [15] and the optimization ability has 
been approved. Therefore QGA is adopted to solve the economic dispatch problem for GTCC units. Simulations were performed by building up models using data from previous literature [16] and optimizing the solutions of the models by QGA. The results show that QGA is quite effective in solving the optimization of economic dispatch problem of GTCC units.

\section{Economic Dispatch Model of GTCC Units}

\subsection{Basic Model of Economic Dispatch Problem}

The economic dispatch problem of GTCC units is to find the optimum combination of units that minimizes the total gas consumption while satisfying the total load demand and operation constraints. In order to analyze the problem through a mathematical model, the total gas consumption of all the GTCC units is described as a function of units' power outputs. And to optimize the solution of the load dispatch problem is to get the value of each unit's power output while the total gas consumption function achieves a minimum. Thus the formulation of a GTCC units load dispatch problem with operation constraints can be described as follows.

$$
\left\{\begin{array}{l}
\min F=\sum_{i=1}^{n} U_{i} \cdot f_{i}\left(P_{i}\right) \\
\text { s.t. } \quad \sum_{i=1}^{n} U_{i} P_{i}=P_{D} \\
P_{i \min } \leq P_{i} \leq P_{i \max } \\
\sum_{i=1}^{n} U_{i} P_{i \max } \geq P_{D}+R
\end{array}\right.
$$

where $\mathrm{F}$ is the objective function corresponding to the total gas consumption (in $\mathrm{m}^{3} / \mathrm{h}$ ); $\mathrm{n}$ is the total number of GTCC units. $f_{i}\left(P_{i}\right)$ is the gas consumption for the ith unit (in $\mathrm{m}^{3} / \mathrm{h}$ ); $P_{i}$ is the power output of unit $\mathrm{i}$ (in MW); $n$ is the number of units in the system; $U_{i}$ represents ith unit's running state and it can only be 0 or 1 which represents stop or running. $P_{D}$ is the system's total demand (in MW); $P_{i \min }$ and $P_{i \max }$ are the lower and upper bounds of power outputs for the ith unit (in MW); $\mathrm{R}$ is the spinning reserve and generally $\mathrm{R}=$ $0.07 P_{D}$ is adopted.

For gas consumption function $f_{i}\left(P_{i}\right)$, binomial expression is usually adopted to fit its characteristic curve as follow.

$$
f_{i}\left(P_{i}\right)=a_{i} P_{i}^{2}+b_{i} P_{i}+c_{i}
$$

where $a_{i}, b_{i}, c_{i}$ are the gas consumption characteristic coefficients of ith unit.

There are some simplified hypotheses of the model: All the $\mathrm{n}$ units can be arranged to start and stop; fuel consumption for start or stop is not considered; line losses are not considered; the fuel property of natural gas is constant; and the total load demand keeps constant within a certain interval.

\subsection{Economic Dispatch Model of GTCC Units Adopting Variable Constraints}

In the model above, $P_{i \min }, P_{i \max }$ are influenced by the performance of a GTCC unit. And the performance of a unit varies with many factors, such as environmental conditions, condenser pressure, inlet and exhaust losses, fuel properties, and so on. Considering environmental factors are always changing with time and location, the influence of environmental conditions is mainly studied to show how the performance of GTCC units varies with environmental conditions.

According to Ref $[17,18]$, for the influence of ambient temperature, generally speaking, every $1^{\circ} \mathrm{C}$ depression in inlet air temperature results in about $0.45 \%$ power increase of GTCC and very slight variation in heat consumption rate [19]. For the influence of barometric pressure, the output and the barometric pressure generally decrease proportionately, but the heat rate and other cycle parameters are not affected. However, for the plant already installed, the variation of this variable is so subtle that can be neglected. For the influence of humid air, the variation is to be too small to be considered.

To calculate the value of $P_{i \min }$ and $P_{i \max }$, correction for ambient temperature needs to be known. The temperature correction factor can be defined as follow.

$$
\varepsilon=\frac{P_{t}}{P_{0}}
$$

where $P_{t}$ is the output power of a GTCC unit under full load operation when the ambient temperature is t. $P_{0}$ is the output power of a GTCC unit under ISO condition $\left(15^{\circ} \mathrm{C}\right)$. Thus, when the ambient temperature is t, the upper bound for power output of the ith unit $P_{i \max }$ can be calculated as follow.

$$
P_{i \max }=\varepsilon P_{0}
$$

Considering the stability and economy of GTCC units, $P_{i \min }=60 \% P_{i \max }$ is usually adopted.

\section{Optimization of Economic Dispatch Problem for GTCC Units Based on Quantum Genetic Algorithm}

\subsection{Quantum Genetic Algorithm}

AjitNarayanan and MarkMoore proposed the concept of Quantum Genetic Algorithm, which is a searching probabilistic method combining the quantum theory and the genetic algorithm. The algorithm is based on some principles of quantum theory such as interference, superposition of states. It adopts quantum bits code to create chromosomes and achieves evolutionary search through 
updating of quantum gates. QCA has the advantages of small population size, strong ability of globally optimization, fast convergent speed and so on.

\subsection{Quantum Code}

QGA uses vector representation to express quantum state, of which the smallest unit of information for representing individuals is quantum bit. Using probability amplitude of quantum bit to represent the codes of chromosome, quantum bit coded chromosome probabilistically represents several states in the search space. The state of a quantum bit can be represented by a superposition of two quantum states as follow [20].

$$
|\psi>=\alpha| 0>+\beta \mid 1>
$$

where $|\psi\rangle,|0\rangle$ and $\mid 1>$ represent respectively the states of a quantum bit; $\alpha$ and $\beta$ are two complex numbers represent the probability amplitudes of the corresponding quantum state. They should satisfy the following equation.

$$
|\alpha|^{2}+|\beta|^{2}=1
$$

where $|\alpha|^{2}$ is the probability to have the value 0 and $|\beta|^{2}$ is the probability to have the value 1 .

The quantum bit can contain information of 0 and 1 simultaneously. Therefore a chromosome containing $\mathrm{m}$ quantum bits can represent $2^{m}$ states.

\subsection{Quantum Gate}

Quantum gate is the actuator of evolution operation. Quantum rotation gate strategy is adopted to achieve population evolution as follow.

$$
\left[\begin{array}{l}
\alpha^{\prime} \\
\beta^{\prime}
\end{array}\right]=\left[\begin{array}{rr}
\cos \theta & -\sin \theta \\
\sin \theta & \cos \theta
\end{array}\right]\left[\begin{array}{l}
\alpha \\
\beta
\end{array}\right]
$$

where $[\alpha, \beta]$ and $\left[\alpha^{\prime}, \beta^{\prime}\right]$ are the probability amplitudes before and after quantum rotation updating. $\theta$ is the rotation angle and its value adopts the methods in paper [21].

\subsection{The Procedure of the Optimization by QGA}

1) Initialize a population $Q(t)$ of $n$ chromosomes randomly and each chromosome contains m quantum bits. Each pair of quantum bit probability amplitudes $\alpha_{i}$ and $\beta_{i}, \mathrm{i}=1,2, \ldots, \mathrm{m}$, are initialized with $1 / \sqrt{2}$, which means the same probability to emerge at the beginning of the algorithm.

2) Observe each chromosome in $\mathrm{Q}(\mathrm{t})$ so that a set of binary solutions $P(t)=\left\{p_{1}^{t}, p_{2}^{t}, p_{3}^{t} \ldots p_{n}^{t}\right\}$ are obtained. Each bit of $p_{j}^{t}, \mathrm{j}=1,2, \ldots, \mathrm{n}$, is formed as follow. Generate a number $\mathrm{r}$ randomly with uniform distribution in range $[0,1]$. If $\mathrm{r}>|\alpha|^{2}$ or $|\beta|^{2}$, the result of the obser- vation will be 1 , otherwise, it will 0 .

3) Measure the fitness of all the solutions observed above. For the load dispatch problem in this paper, the objective function can be described as follow.

$$
g=-\sum_{i=1}^{n}\left[U_{i} \cdot f_{i}\left(P_{i}\right)\right]-C\left(\sum_{i=1}^{n} U_{i} P_{i}-P_{D}\right)^{2}
$$

where $\mathrm{C}$ is a penalty factor which usually takes a large value. In the function above, it can lead to small value of the function if a solution does not satisfy the constraints, so the solution will be deleted. Record the best solution and its fitness.

4) Judge whether the convergence condition is satisfied. If not, the algorithm will use the quantum rotation gate to update the population $\mathrm{Q}(\mathrm{t})$ so that a new population $\mathrm{Q}(\mathrm{t}+1)$ is obtained.

5) The algorithm will go back to step (2) and the process will be repeated iteratively until the convergence condition is satisfied.

\section{Simulation}

In paper [16] the author uses equal micro incremental method to optimize the load dispatch of $3 \times 390 \mathrm{MW}$ gas turbine generation units. In this paper, the gas consumption characteristic equations from paper [16] are used for the calculation of optimal load dispatch by QGA.

According to paper [16] the characteristic coefficient of gas consumption function and bound constraints are shown in Table 1.

Take total load demand =491 MW on July 25, 2007 as an example. The food consistence function is described as follow.

$$
g=-\left[\sum_{i=1}^{3} U_{i} \cdot f_{i}\left(P_{i}\right)+C\left(\sum_{i=1}^{3} U_{i} P_{i}-P_{D}\right)^{2}\right]
$$

The units in operation are just $1 \#$ and $3 \#$, so $\mathrm{U}_{1}=\mathrm{U}_{3}=1, \mathrm{U}_{2}=0$. Take 100 for the penalty factor $\mathrm{C}$.

$$
\left\{\begin{array}{l}
f_{1}\left(P_{1}\right)=0.058427 P_{1}^{2}+117.29 P_{1}+11904 \\
f_{3}\left(P_{3}\right)=0.12681 P_{3}^{2}+75.695 P_{3}+17860
\end{array}\right.
$$

In this QGA, the size of the population is set to 40; the maximum iteration number is 200. Through the optimization of QGA, when the load of 1\# unit is 234.34 MW and $2 \#$ unit is $255.94 \mathrm{MW}$, the maximum value of fitness function reaches-87229.83. The total gas consumption is $85605.81 \mathrm{~m}^{3} \mathrm{~N} / \mathrm{h}$.

Table 1. Gas consumption character coefficients and limit of load.

\begin{tabular}{ccccc}
\hline unit & $\mathbf{a}_{\mathbf{i}}$ & $\mathbf{b}_{\mathbf{i}}$ & $\mathbf{c}_{\mathbf{i}}$ & {$\left[\mathbf{p}_{\min }, \mathbf{p}_{\max }\right]$} \\
\hline 1 & 0.058427 & 117.29 & 11904 & {$[234,390]$} \\
2 & 0.12583 & 75.695 & 18274 & {$[234,390]$} \\
3 & 0.12681 & 71.863 & 17860 & {$[234,390]$} \\
\hline
\end{tabular}




\section{Results and Discussions}

Using the foregoing QGA method to optimize the economic load dispatch problem of GTCC units, the load dispatch results obtained by QGA on October 26, 2007 are compared with those by other methods, as shown in Table 2.

According to the results above, QGA could achieve savings of total gas consumption compared to the equal micro incremental method and AGC instruction. For instance, on October 26, 2007, when the total load demand is $500 \mathrm{MW}$, the load dispatch results of unit $1 \#$ and $3 \#$ are 236.03 MW and 263.25 MW. The gas consumption is $42842.31 \mathrm{~m}^{3} \mathrm{~N} / \mathrm{h}$ and $43609.51 \mathrm{~m}^{3} \mathrm{~N} / \mathrm{h}$ respectively. The total gas consumption is $86451.83 \mathrm{~m}^{3} \mathrm{~N} / \mathrm{h}$, which has a reduction of $2055.50 \mathrm{~m}^{3} \mathrm{~N} / \mathrm{h}$ compared to equal micro incremental method and $1327.151 \mathrm{~m}^{3} \mathrm{~N} / \mathrm{h}$ compared to AGC instruction. It indicates that using QGA to optimize the load dispatch problem of gas turbine units can improve the economic efficiency of the units.

When considering the influence of ambient conditions, according to related literature, it can be assumed that $\varepsilon=0.45 \%$. If the ambient temperature is $25^{\circ} \mathrm{C}$, then the maximum limit of unit i is $P_{i \max }=372.45 \mathrm{MW} \quad i=1,3$, and the minimum limit of unit $\mathrm{i}$ is $P_{i \min }=60 \% P_{i \max }$ $=223.47 \mathrm{MW} i=1,3$. Then the load dispatch results are shown in Table 3.

In Tables 2 and $\mathbf{3}$ the results of load dispatch optimization are compared. It has shown that there are big differences in the results considering the influence of ambient condition or not. For example, when the total load demand is $491 \mathrm{MW}$, the load dispatch results of the model with variable constraints are 223.50 MW and 266.80 MW while the results not considering the influence of ambient temperature are 234.34 MW and 255.94 MW. In fact, considering the influence of ambient conditions can help to make full use of GTCC units' output performance in this situation. The process for QGA to optimize the solution of load dispatch model is shown in Figure 1.

\section{Conclusions}

In this paper, the economic dispatch problem of gas turbine combined-cycle units is proposed and the quantum genetic algorithm is employed on the optimization of the problem.

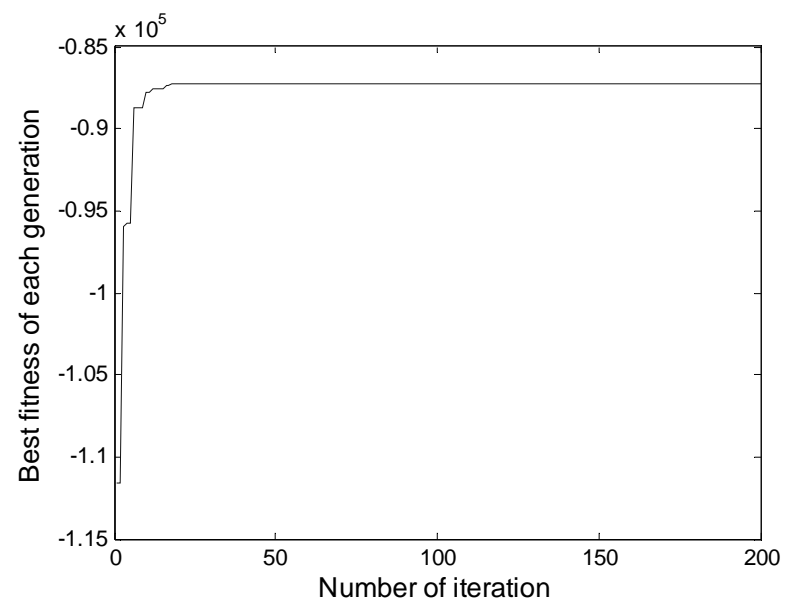

Figure 1. Iterative process of QGA.

Table 2. Load dispatch results of 1\#,3\# by different methods On October 26,2007.

\begin{tabular}{|c|c|c|c|c|c|c|c|}
\hline $\begin{array}{l}\text { Load } \\
\text { MW }\end{array}$ & $\begin{array}{c}\mathbf{P}_{1} \\
\text { (QGA) } \\
\text { MW }\end{array}$ & $\begin{array}{c}\mathbf{P}_{3} \\
\text { (QGA) } \\
\text { MW }\end{array}$ & $\begin{array}{c}\text { 1\# gas consumption } \\
\text { (QGA) } \\
\text { m }^{3} \mathrm{~N} / \mathrm{h}\end{array}$ & $\begin{array}{c}\text { 3\# gas consumption } \\
\text { (QGA) } \\
\mathbf{m}^{3} \mathbf{N} / \mathbf{h}\end{array}$ & $\begin{array}{c}\text { Total gas consumption } \\
\text { (QGA) } \\
\mathbf{m}^{3} \mathrm{~N} / \mathrm{h}\end{array}$ & $\begin{array}{c}\text { Equal micro } \\
\text { incremental method } \\
\mathrm{m}^{3} \mathrm{~N} / \mathrm{h}\end{array}$ & $\begin{array}{c}\text { AGC } \\
\mathbf{m}^{3} \mathbf{N} / \mathbf{h}\end{array}$ \\
\hline 491 & 234.34 & 255.94 & 42598.68 & 43007.13 & 85605.81 & 87114.16 & 88041.39 \\
\hline 500 & 236.03 & 263.25 & 42842.31 & 43609.51 & 86451.83 & 88507.33 & 87778.98 \\
\hline 600 & 287.24 & 312.00 & 50415.61 & 50846.51 & 101262.12 & 103229.10 & 103538.45 \\
\hline 701 & 355.25 & 344.95 & 60945.12 & 58478.96 & 119424.08 & 118737.80 & 119029.83 \\
\hline
\end{tabular}

Table 3. Load dispatch results of 1\#,3\# considering the influence of ambient temperature.

\begin{tabular}{|c|c|c|c|c|c|c|c|}
\hline $\begin{array}{l}\text { Load } \\
\text { MW }\end{array}$ & $\begin{array}{c}P_{1} \\
\text { (QGA) } \\
\text { MW }\end{array}$ & $\begin{array}{c}\mathbf{P}_{3} \\
\text { (QGA) } \\
\text { MW }\end{array}$ & $\begin{array}{c}\text { 1\# gas consumption } \\
(\mathbf{Q G A}) \\
\mathbf{m}^{3} \mathrm{~N} / \mathbf{h}\end{array}$ & $\begin{array}{c}\text { 3\# gas consumption } \\
\text { (QGA) } \\
\mathbf{m}^{3} \mathbf{N} / \mathbf{h}\end{array}$ & $\begin{array}{c}\text { Total gas consumption } \\
\text { (QGA) } \\
\mathbf{m}^{3} \mathrm{~N} / \mathbf{h}\end{array}$ & $\begin{array}{c}\text { Equal micro } \\
\text { incremental method } \\
\mathrm{m}^{3} \mathrm{~N} / \mathrm{h}\end{array}$ & $\begin{array}{c}\text { AGC } \\
\mathbf{m}^{3} \mathbf{N} / \mathbf{h}\end{array}$ \\
\hline 491 & 223.50 & 266.80 & 41412.29 & 47426.59 & 83985.02 & 87114.16 & 88041.39 \\
\hline 500 & 224.56 & 274.72 & 41558.06 & 48565.27 & 84757.70 & 88507.33 & 87778.98 \\
\hline 600 & 287.97 & 311.28 & 50730.95 & 54028.17 & 101366.38 & 103229.10 & 103538.45 \\
\hline 651 & 326.05 & 324.18 & 56697.07 & 56036.98 & 110974.87 & 111009.30 & 111040.91 \\
\hline 701 & 356.62 & 343.58 & 61736.62 & 59135.23 & 119621.05 & 118737.80 & 119029.83 \\
\hline
\end{tabular}


On the basis of analyzing the influence of the ambient conditions on the performance of GTCC units, temperature correction factor is used to improve the economic dispatch model.

The optimization results by QGA are compared with those by equal micro incremental method. Almost all the results obtained by QGA in the simulations are better than other methods used in the literature. With QGA method the gas consumptions are significantly reduced. The searching processes show that QGA has good characteristics of globally optimization, fast convergent speed robustness for initial values and so on.

The comparison of dispatch models for GTCC units considering and not considering the influence of ambient conditions indicates that it is so necessary to adopt variable constraints for the dispatch model of GTCC units.

\section{REFERENCES}

[1] T. S. Kim and S. H. Hwang, "Part Load Performance Analysis of Recuperated Gas Turbines Considering Engine Configuration and Operation Strategy,” Energy, Vol. 31, No. 2-3, 2006, pp. 260-277.

\section{doi:10.1016/j.energy.2005.01.014}

[2] S. H. Yousef and Najjar, "Comparison of Performance for Cogeneration Systems Using Single- or Twin-shaft Gas Turbine Engines,” Applied Thermal Engineering, Vol. 17, No. 2, 1997, pp. 113-124. doi:10.1016/S1359-4311(96)00028-2

[3] B. K. Panigrahi, S. R. Yadav, S. Agrawal and M. K. Tiwari, "A Clonal Algorithm to Solve Economic Load Dispatch,” Electric Power Systems Research, Vol. 77, No. 10, 2007, pp. 1381-1389. doi:10.1016/j.epsr.2006.10.007

[4] W.-M. Lin, F.-S. Cheng, M.-T. Tsay, "An Improved Tabu Search for Economic Dispatch with Multiple Minima," IEEE Transactions on Power System, Vol. 17, No. 1, 2002, pp. 108-112. doi:10.1109/59.982200

[5] C. Yang, Z. L. Yang and R. X. Cai, “Analytical Method For Evaluation of Gas Turbine Inlet Air Cooling in Combined Cycle Power Plant,” Applied Energy, Vol. 86, No. 6, 2009, pp. 848-856.

doi:10.1016/j.apenergy.2008.08.019

[6] C. C. Chuang and D. C. Sue, "Performance Effects of Combined Cycle Power Plant with Variable Condenser Pressure and Loading," Energy, Vol. 30, 2005, pp.1793-1801. doi:10.1016/j.energy.2004.10.003

[7] D. Sanchez, R. Chacartegui, J. M. Munoz, A. Munoz and T. Sanchez, "Performance Analysis of a Heavy Duty Combined Cycle Power Plant Burning Various Syngas Fuels,” International Journal of Hydrogen Energy, Vol. 35, 2010, pp. 337-345.

doi:10.1016/j.ijhydene.2009.10.080

[8] C. L. Chen and S. C. Wang, "Branch and Bound Scheduling for Thermal Generating Units," IEEE Transactions on Energy Conversion, Vol. 8, No. 2, 1993, pp.184-189.
[9] B. H. Chowdhury and S. Rabman, "A Review of Recent 4dvances in Economic Dispatch," IEEE Transactions on Power Systems, Vol. PWRS-5, No. 4, 1990, pp. 1248-1257. doi:10.1109/59.99376

[10] M. Huneault and F. D. Galiana, "Survey of the Optimal Power Flow Literature," IEEE Transactions on Power Systems, Vol. PWRS-6, No. 2, 1991, pp. 762-770. doi:10.1109/59.76723

[11] K. Y. Le, A. Sode-Yome and J. Ho, "Park Adaptive Hopfield Neural Networks for Economic Load Dispatch," IEEE Transactions on Power Systems, Vol. 13, No. 2, 1998.

[12] R. Naresh, J. Dubey, J. Sharma, “Two Phase Neural Network Based Modeling Framework of Constrained Economic Load Dispatch,” IEE Proceedings-Generation Transmission and Distribution, Vol. 151, No. 3, 2004, pp. 373-378.

[13] C. K. Panigrahi, P. K. Chattopadhyay, R. N. Chakrabarti and M. Basu, "Simulated Annealing Technique for Dynamic Economic Dispatch,” Electric Power Components and Systems, Vol. 34, No. 5, 2006, pp. 577-586.

doi:10.1080/15325000500360843

[14] J. E. Lansbeny and L. Wozniak, “Adaptive Hydrogenerator Governor Tuning with a Genetic Algorithm,” IEEE Paper 93 WM 136-2 EC, presented at the IEEERES 1993 Winter Meeting.

[15] L. S. Coelho, R. C. T. De Souza and V. C. Mariani, "Improved Differential Evolution Approach Based on Cultural Algorithm and Diversity Measure Applied to Solve Economic Load Dispatch Problems," Mathematics and Computers Simulation, Vol. 79, No. 10, 2009, pp. 3136-3147. doi:10.1016/j.matcom.2009.03.005

[16] X. G. Peng, C. H. Zhang and X. H. Wang, “Application Research of Optimal Load Dispatch on LNG Power Plant,” Power System Protection and Control, Vol. 38, No. 14, 2010, pp. 84-87.

[17] F. R. P. Arrieta and E. E. S. Lora, "Influence of Ambient Temperature on Combined-cycle Power-plant Performance,” Applied Energy, Vol. 80, No. 3, 2005, pp. 261-272. doi:10.1016/j.apenergy.2004.04.007

[18] R. Hosseini, A. Beshkani and M. Soltani, "Performance Improvement of Gas Turbines of Fars (Iran) Combined Cycle Power Plant by Intake Air Cooling Using a Media Evaporative Cooler," Energy Conversion and Management, Vol. 48, No. 4, 2007, pp. 1055-1064.

doi:10.1016/j.enconman.2006.10.015

[19] C. Yang, Z. L. Yang and R. X. Cai, “Analytical Method For Evaluation of Gas Turbine Inlet air Cooling in Combined Cycle Power Plant,” Applied Energy, Vol. 86, No. 6, 2009, pp. 848-856.

doi:10.1016/j.apenergy.2008.08.019

[20] J. X. Vianna, N. D. L. de A. Bernert and L. dos S. Coelho, "Improved Quantum-inspired Evolutionary Algorithm with Diversity Information Applied to Economic Dispatch Problem with Prohibited Operating Zones,” Energy Conversion and Management, Vol. 52, No. 1, 2011,pp. 8-14.doi:10.1016/j.enconman.2010.05.023 
[21] K.-H. Han and J.-H. Kim, "Quantum-inspired Evolutionary Algorithm for a Class of Combinatorial Optimization," IEEE Transactions on Evolutionary
Computation, Vol. 6, No. 6, 2002, pp. 580-593. doi:10.1109/TEVC.2002.804320 